\title{
О ПОЛНОМ ОПРЕДЕЛЕНИИ ОБЪЕМНОГО НАПРЯЖЕННОГО СОСТОЯНИЯ МЕТОДОМ ФОТОУПРУГОСТИ
}

\author{
Х. АБЕН, \\ кандидат технических наук
}

В настоящее время известно несколько методов полного определения объемного капряженного состояния фотоупругих моделей. Из них только обобщенный метод разности касательных напряжений, предложенный впервые В. М. Красновым ['], может быть эффективно применен в общем случае напряженного состояния. Метод измерения линейной деформации при размораживании напряжений, предложенный А. Куске [2], отказывает, когда коэффициент Пуассона равен или близок к 1/2 (см. [ $\left.{ }^{3}\right]$ ). Интегрирование обобщенных уравнений Ляме-Максвелла, выведенных Х. Джессопом [4], практически применимо только в случаях пересечения двух плоскостей симметрии и осесимметричной задачи. Метод, предложенный X. Раке [5], позволяет определять напряжения лишь в отдельных, заранее определенных точках, и, кроме того, он сложен с точки зрения техники эксперимента. Интересный чисто поляризационно-оптический метод предложен Р. Плехата [ $\left.{ }^{6}\right]$, однако он требует столь высокой точности эксперимента, что применение его в практике становится затруднительным. Все это свидетельствует о том, что проблема разработки новых методов полного определения объемного напряженного состояния является весьма актуальной.

В настоящей статье рассматривается полное определение объемного напряженного состояния в замороженных срезах чисто поляризационно-оптическим методом, использующим наклонное просвечивание вместе с измерением абсолютных разностей хода. Выводятся основные формулы метода, причем рассматривается также применение универсального метода Е. С. Федорова. Анализируется точность предлагаемого метода.

Отметим, что предложение об измерении абсолютных разностей хода при исследовании объемного напряженного состояния внесено Б. М. Зуевым [1"].

\section{1. Применение обычного полярископа}

Будем рассматривать вырезанный из замороженной модели срез. Главные напряжения произвольно ориентированы относительно плоскости среза, на которой находятся оси $x$ и $y$ прямоугольной правой системы координат $x, y, z$.

Производя нормальное и два наклонных просвечивания среза с одновременным определением разностей хода и параметров квазиизоклин, можно определить пять независимых компонентов напряжения [7]. Если повороты среза для наклонного просвечивания производятся около оси $x$ на угол $\Theta_{x}$ и около оси $y$ на угол $\Theta_{y}$, то из формул, выведенных в [7], получим: *

* Угол $\Theta_{x}$ считается положительным, если положительная ветвь оси $y$ поворачивается в направлении положнтельной ветви оси $z ; \Theta_{y}$ считается положительным, если положительная ветвь оси $\boldsymbol{z}$ поворачивается в направлении пөложительной ветви оси $x$. 


$$
\begin{aligned}
\sigma_{x}-\sigma_{y} & =\delta_{\mathrm{H}} \cos 2 \varphi_{\mathrm{H}} \\
\tau_{x y} & =\frac{1}{2} \delta_{\mathrm{H}} \sin 2 \varphi_{\mathrm{H}} \\
\tau_{z x} & =\frac{1}{2} \operatorname{ctg} \Theta_{x}\left(\delta_{\mathrm{H}} \sin 2 \varphi_{\mathrm{H}}-\delta_{x} \sin 2 \varphi_{x}\right) \\
\tau_{y z} & =\frac{1}{2} \operatorname{ctg} \Theta_{y}\left(-\delta_{\mathrm{H}} \sin 2 \varphi_{\mathrm{H}}+\delta_{y} \sin 2 \varphi_{y}\right) \\
\sigma_{y}-\sigma_{z} & =\frac{1}{\sin ^{2} \Theta_{x}}\left[\delta_{x} \cos \Theta_{x} \cos 2 \varphi_{x}-\delta_{\mathrm{H}} \cos 2 \varphi_{\mathrm{H}}+\right. \\
& \left.+\frac{1}{2} \operatorname{ctg} \Theta_{y} \sin 2 \Theta_{x}\left(\delta_{\mathrm{H}} \sin 2 \varphi_{\mathrm{H}}-\delta_{y} \sin 2 \varphi_{y}\right)\right]
\end{aligned}
$$

Если же для наклонного просвечивания повороты среза производятся около оси $x$ на разные углы $\Theta_{x}$ и $\Theta_{x}{ }^{\prime}$, то

$$
\begin{gathered}
\tau_{y z}=\frac{1}{A}\left[\sin ^{2} \Theta_{x}\left(\delta_{x}^{\prime} \cos \Theta_{x}^{\prime} \cos 2 \varphi_{x}^{\prime}-\delta_{\mathrm{H}} \cos 2 \varphi_{\mathrm{H}}\right)-\sin ^{2} \Theta_{x}^{\prime}\left(\delta_{x} \cos \Theta_{x} \cos 2 \varphi_{x}-\right.\right. \\
\left.\left.-\delta_{\mathrm{H}} \cos 2 \varphi_{\mathrm{H}}\right)\right]
\end{gathered}
$$

$\sigma_{y}-\sigma_{z}=\frac{1}{A}\left[\sin 2 \Theta_{x}^{\prime}\left(\delta_{x} \cos \Theta_{x} \cos 2 \varphi_{x}-\delta_{\mathrm{H}} \cos 2 \varphi_{\mathrm{H}}\right)-\sin 2 \Theta_{x}\left(\delta_{x}^{\prime} \cos \Theta_{x}^{\prime} \cos 2 \varphi_{x}^{\prime}-\right.\right.$

$$
\left.\left.-\delta_{\mathrm{H}} \cos 2 \varphi_{\text {H. }}\right)\right]
$$

где

$$
A=\sin ^{2} \Theta_{x} \sin 2 \Theta_{x}^{\prime}-\sin ^{2} \Theta_{x}^{\prime} \sin 2 \Theta_{x}
$$

Здесь приняты следующие обозначения: $\delta_{i}=\bar{\delta}_{i} / C t, \bar{\delta}_{i}-$ разности хода при нормальном и наклонных просвечиваниях, $C-$ оптическая постоянная, $t$ - толщина среза, $\varphi_{i}$ - параметры квазиизоклин *.

Пусть при нормальном просвечивании среза измеряются абсолютные разности хода $\delta_{1}$ и $\delta_{2}$, соответствующие квазиглавным напряжениям $\sigma^{\prime}{ }_{1}$ и $\sigma_{2}^{\prime}$. Тогда получим следующие соотношения:

$$
\begin{aligned}
& \delta_{1}=C \sigma_{1}^{\prime}+C_{1}\left(\sigma_{x}+\sigma_{y}+\sigma_{z}\right) \\
& \delta_{2}=C \sigma_{2}^{\prime}+C_{1}\left(\sigma_{x}+\sigma_{y}+\sigma_{z}\right)
\end{aligned}
$$

где $C_{1}-$ вторая оптическая постоянная.

На основе соотношения

$$
\sigma_{1}^{\prime}+\sigma_{2}^{\prime}=\sigma_{x}+\sigma_{y}
$$

получим из (9) и (10) уравнение

$$
K_{2} \sigma_{x}+K_{2} \sigma_{y}+2 \sigma_{z}=\delta^{*}
$$

где приняты обозначения

$$
K_{1}=\frac{C}{C_{1}}, \quad K_{2}=K_{1}+2, \quad \delta^{*}=\frac{\delta_{1}+\delta_{2}}{C_{1}}
$$

* Углы $\varphi_{i}$ считаются от положительного направления оси $x$ до направления алгебраически большего квазиглавного напряжения - при повороте около оси $x$, и на ось, $x^{\prime}\left(x^{\prime} \perp y, \angle x x^{\prime}=\Theta_{y}\right)-$ при повороте около оси $y$.

Индекс $\boldsymbol{H}$ обозначает нормальное просвечивание; индексы $x$ и $y$ указывают на ось, около которой поворачивается модель для наклонного просвечивания. 
Обозначая правые части выражения (1) через $F_{1}$ и выражений (5) и (7) через $F_{2}$, имеем следующую систему уравнений для определения нормальных напряжений:

$$
\left.\begin{array}{rl}
K_{2} \sigma_{x}+K_{2} \sigma_{y}+2 \sigma_{z} & =\delta^{*} \\
\sigma_{x}-\sigma_{y} & =F_{1} \\
\sigma_{y}-\sigma_{z} & =F_{2}
\end{array}\right\}
$$

из которой нормальные напряжения определяются формулами

$$
\begin{aligned}
\sigma_{x} & =\frac{1}{2\left(1+K_{2}\right)}\left[\delta^{*}+\left(2+K_{2}\right) F_{1}+2 F_{2}\right] \\
\sigma_{y} & =\frac{1}{2\left(1+K_{2}\right)}\left(\delta^{*}-K_{2} F_{1}+2 F_{2}\right) \\
\sigma_{z} & =\frac{1}{2\left(1+K_{2}\right)}\left(\delta^{*}-K_{2} F_{1}-2 K_{2} F_{2}\right)
\end{aligned}
$$

Таким образом, все шесть компонентов напряжения определены. Отметим, что для определения пяти компонентов напряжения можно вместо метода наклонного просвечивания применять также методику, использующую нормальное просвечивание срезов и субсрезов [3].

\section{2. Применение универсального метода Е. С. Федорова}

Применяя при поляризационно-оптических исследованиях универсальный метод Е. С. Федорова, можно определять направляющие косинусы направлений главных напряжений, обозначение которых дано в табл. 1 .

Таблица 1

\begin{tabular}{c|c|c|c}
\hline & $x$ & $y$ & $z$ \\
\hline$\sigma_{1}$ & $l_{1}$ & $m_{1}$ & $n_{1}$ \\
\hline$\sigma_{2}$ & $l_{2}$ & $m_{2}$ & $n_{2}$ \\
\hline$\sigma_{3}$ & $l_{3}$ & $m_{3}$ & $n_{3}$
\end{tabular}

Определяя при произвольном просвечивании разность хода и параметр квазиизоклины, получим два соотношения между разностями главных напряжений [7]:

$$
\begin{aligned}
& \delta_{x} \cos \Theta_{x} \cos 2 \varphi_{x}=\left(\sigma_{j}-\sigma_{k}\right)\left[l_{j}^{2}-\left(m_{j} \cos \Theta_{x}-n_{j} \sin \Theta_{x}\right)^{2}\right]- \\
& -\left(\sigma_{k}-\sigma_{i}\right)\left[l_{i}^{2}-\left(m_{i} \cos \Theta_{x}-n_{i} \sin \Theta_{x}\right)^{2}\right] \\
& \frac{1}{2} \delta_{x} \cos \Theta_{x} \sin 2 \varphi_{x}=\left(\sigma_{j}-\sigma_{k}\right) l_{j}\left(m_{j} \cos \Theta_{x}-n_{j} \sin \Theta_{x}\right)- \\
& -\left(\sigma_{k}-\sigma_{i}\right) l_{i}\left(m_{i} \cos \Theta_{x}-n_{i} \sin \Theta_{x}\right)
\end{aligned}
$$

Легко доказать, что в общем случае уравнения (18) и (19) не являются линейно зависимыми. Действительно, если бы уравнения (18) и (19) были линейно зависимыми, то должно быть выполнено условие 


$$
\frac{1}{2} \operatorname{tg} 2 \varphi_{x}=\frac{l_{j}\left(m_{j} \cos \Theta_{x}-n_{j} \sin \Theta_{x}\right)}{l_{j}^{2}-\left(m_{j} \cos \Theta_{x}-n_{j} \sin \Theta_{x}\right)^{2}}=\frac{l_{i}\left(m_{i} \cos \Theta_{x}-n_{i} \sin \Theta_{x}\right)}{l_{i}^{2}-\left(m_{i} \cos \Theta_{x}-n_{i} \sin \Theta_{x}\right)^{2}}
$$

Согласно формуле (20), параметр квазиизоклины должен зависеть только от ориентации главных осей оптического эллипсоида относительно световой нормали. Вместе с тем известно, что параметр квазиизоклины зависит также от величины главных напряжений. Поэтому соотношение (20) не имеет места и уравнения (18) и (19) линейно независимы. Следовательно, производя только одно просвечивание среза, можно из (18) и (19) определить разности главных напряжений:

$$
\sigma_{j}-\sigma_{k}=\frac{B_{j, k}}{B}
$$

где

$$
\begin{gathered}
B=l_{j}\left(m_{j} \cos \Theta_{x}-n_{j} \sin \Theta_{x}\right)\left[l_{i}^{2}-\left(m_{i} \cos \Theta_{x}-n_{i} \sin \Theta_{x}\right)^{2}\right]- \\
-l_{i}\left(m_{i} \cos \Theta_{x}-n_{i} \sin \Theta_{x}\right)\left[l_{j}^{2}-\left(m_{j} \cos \Theta_{x}-n_{j} \sin \Theta_{x}\right)^{2}\right] \\
B_{j, k}=\frac{1}{2} \delta_{x} \cos \Theta_{x} \sin 2 \varphi_{x}\left[l_{i}^{2}-\left(m_{i} \cos \Theta_{x}-n_{i} \sin \Theta_{x}\right)^{2}\right]- \\
-\delta_{x} \cos \Theta_{x} \cos 2 \varphi_{x} l_{i}\left(m_{i} \cos \Theta_{x}-n_{i} \sin \Theta_{x}\right)
\end{gathered}
$$

Для нормального просвечивания имеем из (22) и (23)

$$
\begin{aligned}
B & =l_{j} m_{j}\left(l_{i}^{2}-m_{i}^{2}\right)-l_{i} m_{i}\left(l_{j}^{2}-m_{j}^{2}\right) \\
B_{j, k} & =\delta_{\mathrm{H}}\left[\frac{1}{2}\left(l_{i}^{2}-m_{i}^{2}\right) \sin 2 \varphi_{\mathrm{H}}-l_{t} m_{i} \cos 2 \varphi_{\mathrm{H}}\right]
\end{aligned}
$$

Только в случае, когда одно (или два) из главных напряжений находится в плоскости, перпендикулярной лучу, формулы (21) - (25) неприменимы, так как тогда условие (20) выполняется тождественно. Это легко доказать для случая нормального просвечивания среза. Принимая $\Theta_{x}=0$, получим из (20)

$$
\operatorname{tg} 2 \varphi_{\mathrm{H}}=\frac{2 l_{j} m_{j}}{l_{j}^{2}-m_{j}^{2}}
$$

Если главное напряжение $\sigma_{j}$ находится в плоскости среза, то

$$
\begin{aligned}
l_{j} & =\cos \varphi_{н} \\
m_{j} & =\sin \varphi_{н}
\end{aligned}
$$

На основе (27) и (28) условие (26) выполняется тождественно.

Выбирая подходящий угол $\Theta_{x}$, можно срез всегда просвечивать в направлении, которое не является перпендикулярным ни одному из главных напряжений. Для повышения точности желательно, чтобы направление просвечивания составляло с направлениями главных напряжений по возможности равные углы.

Как известно, применяя метод Е. С. Федорова, можно разности главных напряжений определять также измерением разностей хода в двух главных плоскостях оптического эллипсоида $\left.{ }^{8}\right]$ или измерением одной главной разности хода и угла между оптическими осями [9]. Так 
как при исследовании фотоупругих моделей приходится практически ограничиваться поворотами среза в пределах $45^{\circ}$ от положения нормального просвечивания, то непосредственное измерение двух главных разностей хода удается редко. Если оптические оси находятся вблизи плоскости среза, то невозможно также определить угол между ними. Поэтому метод определения разностей главных напряжений, о котором говорилось выше, является более простым и общим.

При использовании изложенного метода вопрос о том, какие именно главные напряжения направлены по определенным осям оптического эллипсоида, решается просто. Наибольшая разность главных напряжений определяется формулой

$$
\sigma_{1}-\sigma_{3}=\max \left|\sigma_{i}-\sigma_{j}\right|
$$

Если $\max \left(\sigma_{i}-\sigma_{j}\right)$ является положительной, то $i=1$ и $j=3$, если же $\max \left(\sigma_{i}-\sigma_{j}\right)$ отрицательна, то $i=3$ и $j=1$.

Измеряя при нормальном просвечивании абсолютные разности хода, получим соотношения (9) и (10), которые запишем в виде

$$
\begin{aligned}
& \delta_{1}=C \sigma_{1}^{\prime}+C_{1}\left(\sigma_{i}+\sigma_{j}+\sigma_{k}\right) \\
& \delta_{2}=C \sigma_{2}^{\prime}+C_{1}\left(\sigma_{i}+\sigma_{i}+\sigma_{k}\right)
\end{aligned}
$$

Из (30) и (31) получим следующее уравнение:

$$
\begin{gathered}
\sigma_{i}\left[K_{1}\left(l_{i}^{2}+m_{i}^{2}\right)+2\right]+\sigma_{j}\left[K_{1}\left(l_{j}^{2}+m_{j}^{2}\right)+2\right]+ \\
+\sigma_{k}\left[K_{1}\left(l_{k}^{2}+m_{k}^{2}\right)+2\right]=\delta^{*}
\end{gathered}
$$

Уравнения (18) и (19) запишем в преобразованном виде:

$$
\begin{gathered}
\sigma_{i}\left[l_{i}^{2}-\left(m_{i} \cos \Theta_{x}-n_{i} \sin \Theta_{x}\right)^{2}\right]+\sigma_{j}\left[l_{j}^{2}-\left(m_{j} \cos \Theta_{x}-n_{j} \sin \Theta_{x}\right)^{2}\right]+ \\
+\sigma_{k}\left[l_{k}^{2}-\left(m_{k} \cos \Theta_{x}-n_{k} \sin \Theta_{x}\right)^{2}\right]=\delta_{x} \cos \Theta_{x} \cos 2 \varphi_{x} \\
\sigma_{i} l_{i}\left(m_{i} \cos \Theta_{x}-n_{i} \sin \Theta_{x}\right)+\sigma_{i} l_{j}\left(m_{j} \cos \Theta_{x}-n_{i} \sin \Theta_{x}\right)+ \\
+\sigma_{k} l_{k}\left(m_{k} \cos \Theta_{x}-n_{k} \sin \Theta_{x}\right)=\frac{1}{2} \delta_{x} \cos \Theta_{x} \sin 2 \varphi_{x}
\end{gathered}
$$

Из системы уравнений (32) - (34) главные напряжения определяются формулами

где

$$
\sigma_{i}=\frac{D_{i}}{D}
$$

$$
\begin{gathered}
D=\left[l_{i}^{2}-\left(m_{i} \cos \Theta_{x}-n_{i} \sin \Theta_{x}\right)^{2}\right] \times \\
\times\left\{l_{j}\left(m_{j} \cos \Theta_{x}-n_{j} \sin \Theta_{x}\right)\left[K_{1}\left(l_{k}^{2}+m_{k}^{2}\right)+2\right]-\right. \\
\left.-l_{k}\left(m_{k} \cos \Theta_{x}-n_{k} \sin \Theta_{x}\right)\left[K_{1}\left(l_{j}^{2}+m_{\cdot j}^{2}\right)+2\right]\right\}+ \\
+\left[l_{j}^{2}-\left(m_{j} \cos \Theta_{x}-n_{j} \sin \Theta_{x}\right)^{2}\right] \times \\
\times\left\{l_{k}\left(m_{k} \cos \Theta_{x}-n_{k} \sin \Theta_{x}\right)\left[K_{1}\left(l_{i}^{2}+m_{i}^{2}\right)+2\right]-\right. \\
\left.-l_{i}\left(m_{i} \cos \Theta_{x}-n_{i} \sin \Theta_{x}\right)\left[K_{1}\left(l_{k}^{2}+m_{k}^{2}\right)+2\right]\right\}+ \\
\quad+\left[l_{k}^{2}-\left(m_{k} \cos \Theta_{x}-n_{k} \sin \Theta_{x}\right)^{2}\right] \times \\
\times\left\{l_{i}\left(m_{i} \cos \Theta_{x}-n_{i} \sin \Theta_{x}\right)\left[K_{1}\left(l_{j}^{2}+m_{j}^{2}\right)+2\right]-\right. \\
\left.-l_{j}\left(m_{j} \cos \Theta_{x}-n_{j} \sin \Theta_{x}\right)\left[K_{1}\left(l_{i}^{2}+m_{i}^{2}\right)+2\right]\right\}
\end{gathered}
$$


$D_{i}=\delta_{x} \cos \Theta_{x} \cos 2 \varphi_{x}\left\{l_{j}\left(m_{j} \cos \Theta_{x}-n_{j} \sin \Theta_{x}\right)\left[K_{1}\left(l_{k}^{2}+m_{k}^{2}\right)+2\right]-\right.$

$\left.-l_{k}\left(m_{k} \cos \Theta_{x}-n_{k} \sin \Theta_{x}\right)\left[K_{1}\left(l_{j}^{2}+m_{j}^{2}\right)+2\right]\right\}+$

$+\frac{1}{2} \delta_{x} \cos \Theta_{x} \sin 2 \varphi_{x}\left\{\left[K_{1}\left(l_{j}^{2}+m_{j}^{2}\right)+2\right]\left[l_{k}^{2}-\left(m_{k} \cos \Theta_{x}-n_{k} \sin \Theta_{x}\right)^{2}\right]-\right.$ $\left.-\left[K_{1}\left(l_{k}^{2}+m_{k}^{2}\right)+2\right]\left[l_{j}^{2}-\left(m_{j} \cos \Theta_{x}-n_{j} \sin \Theta_{x}\right)^{2}\right]\right\}+$

$+\delta^{*}\left\{l_{k}\left(m_{k} \cos \Theta_{x}-n_{k} \sin \Theta_{x}\right)\left[l_{j}^{2}-\left(m_{j} \cos \Theta_{x}-n_{j} \sin \Theta_{x}\right)^{2}\right]-\right.$

$\left.-l_{j}\left(m_{j} \cos \Theta_{x}-n_{j} \sin \Theta_{x}\right)\left[l_{k}^{2}-\left(m_{k} \cos \Theta_{x}-n_{k} \sin \Theta_{x}\right)^{2}\right]\right\}$

Для случая нормального просвечивания имеем из (36) и (37):

$D=\left(l_{i}^{2}-m_{i}^{2}\right)\left\{\left[K_{1}\left(l_{k}^{2}+m_{k}^{2}\right)+2\right] l_{j} m_{j}-\left[K_{1}\left(l_{j}^{2}+m_{j}^{2}\right)+2\right] l_{k} m_{k}\right\}+$

$+\left(l_{j}^{2}-m_{j}^{2}\right)\left\{\left[K_{1}\left(l_{i}^{2}+m_{i}^{2}\right)+2\right] l_{k} m_{k}-\left[K_{1}\left(l_{k}^{2}+m_{k}^{2}\right)+2\right] l_{i} m_{i}\right\}+$

$+\left(l_{k}^{2}-m_{k}^{2}\right)\left\{\left[K_{1}\left(l_{j}^{2}+m_{j}^{2}\right)+2\right] l_{i} m_{i}-\left[K_{1}\left(l_{i}^{2}+m_{i}^{2}\right)+2\right] l_{j} m_{j}\right\}$

$D_{i}=\delta_{\text {н }} \cos 2 \varphi_{\text {н }}\left\{\left[K_{1}\left(l_{k}^{2}+m_{k}^{2}\right)+2\right] l_{j} m_{j}-\left[K_{1}\left(l_{j}^{2}+m_{j}^{2}\right)+2\right] l_{k} m_{k}\right\}+$ $+\frac{1}{2} \delta_{\mathrm{H}}-\operatorname{in} 2 \varphi_{\mathrm{H}}\left\{\left[K_{1}\left(l_{j}^{2}+m_{j}^{2}\right)+2\right]\left(l_{k}^{2}-m_{k}^{2}\right)-\left[K_{1}\left(l_{k}^{2}+m_{k}^{2}\right)+2\right]\left(l_{j}^{2}-m_{j}^{2}\right)\right\}+$

$$
+\delta^{*}\left[\left(l_{j}^{2}-m_{j}^{2}\right) l_{k} m_{k}-\left(l_{k}^{2}-m_{k}^{2}\right) l_{j} m_{j}\right]
$$

\section{3. Два главных напряжения находятся в плоскости среза}

Если плоскость среза совпадает с плоскостью главных напряжений $\sigma_{i}, \sigma_{j}$, то необходимо производить нормальное и одно наклонное просвечивания среза [7]. Имеем

$$
\begin{aligned}
\sigma_{i}-\sigma_{j}=\delta_{\mathrm{H}} \\
\sigma_{i}-\sigma_{k}=\frac{1}{\sin ^{2} \Theta_{x}}\left[\delta_{x} \cos \Theta_{x} \cos 2 \varphi_{x}+\delta_{\mathrm{H}}\left(\sin ^{2} \varphi_{\mathrm{H}}-\cos ^{2} \varphi_{\mathrm{H}} \cos ^{2} \Theta_{x}\right)\right] \\
\sigma_{j}-\sigma_{k}=\frac{1}{\sin ^{2} \Theta_{x}}\left[\delta_{x} \cos \Theta_{x} \cos 2 \varphi_{x}-\delta_{\mathrm{H}}\left(\cos ^{2} \varphi_{\mathrm{H}}-\sin ^{2} \varphi_{\mathrm{H}} \cos ^{2} \Theta_{x}\right)\right]
\end{aligned}
$$

Измерение абсолютных разностей хода при нормальном просвечивании дает

$$
\begin{aligned}
& \delta_{1}=C \sigma_{i}+C_{1}\left(\sigma_{i}+\sigma_{i}+\sigma_{k}\right) \\
& \delta_{2}=C \sigma_{i}+C_{1}\left(\sigma_{i}+\sigma_{i}+\sigma_{k}\right)
\end{aligned}
$$

Из (43) и (44) получим уравнение

$$
K_{2} \sigma_{i}+K_{2} \sigma_{j}+2 \sigma_{k}=\delta^{*}
$$

Из системы (41), (42) и (45) главные напряжения определяются формулами

$$
\begin{aligned}
\sigma_{i} & =\frac{1}{2\left(1+K_{2}\right)}\left[\delta^{*}+\left(2+K_{2}\right) G_{1}+2 G_{2}\right] \\
\sigma_{j} & =\frac{1}{2\left(1+K_{2}\right)}\left(\delta^{*}-K_{2} G_{1}+2 G_{2}\right) \\
\sigma_{k} & =\frac{1}{2\left(1+K_{2}\right)}\left(\delta^{*}-K_{2} G_{1}-2 K_{1} G_{2}\right)
\end{aligned}
$$

где $G_{1}$ и $G_{2}$ обозначают соответственно правые стороны выражений (40) и (42). 


\section{4. Анализ точности метода}

Теоретически изложенный метод позволяет полностью определять объемное напряженное состояние. Однако выведенные формулы включают множество экспериментальных данных, определение которых связано с экспериментальными ошибками. Поэтому представляет интерес выяснить, какую точность можно ожидать при определении напряжений. Чтобы полностью ответить на этот вопрос, необходимо вычислить ожидаемые ошибки компонентов напряжения для наиболее характерных случаев напряженного состояния, предполагая при этом некоторую точность эксперимента. Такой анализ различных методов с точки зрения их точности для случая применения наклонного просвечивания при исследовании плоской задачи был приведен в работе [7]. Для объемного напряженного состояния это сделать гораздо сложнее, так как число различных характерных напряженных состояний больше и сами вычисления также более громоздки. Поэтому ограничимся некоторыми общими соображениями и приведем один численный пример.

Если экспериментальные ошибки не слишком велики, то предельная абсолютная ошибка $\Delta \sigma_{i}$ некоторого компонента напряжения $\sigma_{i}$ выражается в виде

$$
\Delta \sigma_{i}=\sum_{j}\left|\frac{\partial \sigma_{i}}{\partial \Theta_{j}}\right| \Delta \Theta_{j}+\sum_{j}\left|\frac{\partial \sigma_{i}}{\partial \delta_{j}}\right| \Delta \delta_{j}+\sum_{j}\left|\frac{\partial \sigma_{i}}{\partial \varphi_{j}}\right| \Delta \varphi_{i}+\left|\frac{\partial \sigma_{i}}{\partial \delta^{*}}\right| \Delta \delta^{*}
$$

В случае применения метода Е. С. Федорова имеем

$$
\begin{gathered}
\Delta \sigma_{i}=\left|\frac{\partial \sigma_{i}}{\partial \delta_{x}}\right| \Delta \delta_{x}+\left|\frac{\partial \sigma_{i}}{\partial \varphi_{x}}\right| \Delta \varphi_{x}+\sum_{j}\left|\frac{\partial \sigma_{i}}{\partial l_{j}}\right| \Delta l_{j}+ \\
+\sum_{j}\left|\frac{\partial \sigma_{i}}{\partial m_{j}}\right| \Delta m_{j}+\sum_{j}\left|\frac{\partial \sigma_{i}}{\partial n_{j}}\right| \Delta n_{j}+\left|\frac{\partial \sigma_{i}}{\partial \delta^{*}}\right| \Delta \delta^{*}
\end{gathered}
$$

где $\Delta \Theta_{j}, \Delta \delta_{j}, \Delta \varphi_{j}, \Delta \delta^{*}, \Delta l_{j}, \Delta m_{j}, \Delta n_{j}$ - предельные абсолютные ошибки соответствующих экспериментальных величин. На основе (49), (50) и выведенных выше формул можно выписать выражения для предельных абсолютных ошибок вычисляемых компонентов напряжения. Из-за громоздкости этих выражений мы их не приводим.

Предельная абсолютная ошибка недостаточно хорошо характеризует точность метода, так как вероятность возникновения такой ошибки ничтожна. Гораздо лучше ожидаемая точность характеризуется средней квадратичной ошибкой.

Из теории ошибок известно, что если величина̄ $E$ является линейной функцией независимых величин $E_{i}$

$$
E=\Sigma e_{i} E_{i}
$$

где $e_{i}$ - постоянные, то средняя квадратичная ошибка $\varepsilon$ величины $E$ определяется формулой

$$
\varepsilon= \pm \sqrt{\Sigma e_{i}^{2} \varepsilon_{i}^{2}}
$$

где $\varepsilon_{i}-$ средние квадратичные ошибки величин $E_{i}$.

В выражениях предельных ошибок компонентов напряжения ошибки отдельных экспериментальных данных можно считать независимыми, 
так как ошибка каждой экспериментальной величины обусловлена в основном неточностью определения этой самой величины *. Поэтому изложенный выше метод можно использовать для определения средней квадратичной ошибки величины $\Delta \sigma_{i}$, а следовательно, и величины $\sigma_{i}$. Для этого необходимо знать среднюю квадратичную ошибку $\varepsilon_{i}$ отдельных экспериментальных данных. Предположим, что экспериментальные ошибки подчиняются нормальному распределению. В этом случае можно применять

$$
\varepsilon_{i}=\frac{1}{3} \bar{\Delta}_{i}
$$

где $\bar{\Delta}_{i}$ - максимальные вероятные абсолютные ошибки экспериментальных данных. На основе устойчивости нормального закона максимальная вероятная абсолютная ошибка величины $E$ выражается в виде

$$
\bar{\Delta} E=3 \varepsilon
$$

Изложим некоторые общие соображения относительно ожидаемой точности предложенного метода. Из выражений (1) - (7) и (15) (17) видно, что ошибки компонентов напряжения не могут стать бесконечно большими. Действительно, в знаменателях выражений ошибок не будет членов, которые могли бы равняться нулю, а в числителях не будет членов, равных бесконечности.

Хотя высказанное заключение может показаться тривиальным, оно имеет существенное значение. Анализ, проведенный в работе [7], показал, что почти у всех методов наклонного просвечивания, применяемых при решении плоской задачи, ошибки при некоторых напряженных состояниях бесконечно велики. Такие методы в общем случае мало пригодны для практики. В названной работе было также выяснено, что единственным методом наклонного просвечивания для исследования плоского напряженного состояния, у которого ошибки никогда не бывают бесконечно или чрезмерно большими, является метод, использующий при каждом просвечивании как разность хода, так и параметр квазиизоклины. Формулы (1) - (7) по существу представляют собой обобщение этого метода на объемное напряженное состояние. Можно полагать, что свойства метода в некоторой мере сохраняются и в случае объемного напряженного состояния.

Из (1) - (5) видно, что ошибки тем меньше, чем больше углы поворота модели. Если оба поворота модели производятся около оси $x$ на разные углы, то из (6) и (7) следует, что в знаменатель выражений ошибок входит величина $A$. Чем больше $A$, тем меньше ошибки. Следовательно, желательно поворачивать модель для наклонного просвечивания в разные стороны. Наиболее выгодно использовать $\Theta_{x}=45^{\circ}$, $\boldsymbol{\theta}^{\prime}{ }_{x}=-45^{\circ}$, так как в этом случае $A$ принимает максимальную абсолютную величину: $|A|=1$.

В случае применения метода Е. С. Федорова в знаменатель выражений ошибок разностей главных напряжений входит величина $B$ и в знаменатель выражений ошибок главных напряжений - величина $D$. Подходящим выбором угла $\Theta_{x}$ можно всегда достичь того, что $B$ и $D$ не будут равны нулю. Таким образом, при применении универсального метода Е. С. Федорова величина ошибок также ограничена.

- Приходится учитывать, что в выражении (50) только три из направляющих конусов являются независимыми. 
При мер. Рассмотрим следующее напряженное состояние: $\sigma_{1}=3, \sigma_{2}=1$, $\sigma_{3}=-1$. Направляющие косинусы даны в табл. 2.

Таблица 2

\begin{tabular}{c|c|c|c}
\hline & $x$ & $y$ & $z$ \\
\hline$\sigma_{1}$ & 0,500 & 0,500 & 0,707 \\
\hline$\sigma_{2}$ & $-0,289$ & 0,866 & $-0,425$ \\
\hline$\sigma_{3}$ & 0,816 & 0 & $-0,565$
\end{tabular}

В данном случае имеем

$$
\begin{aligned}
\sigma_{x}=0,17 ; & \sigma_{y}=1,50 ; & \sigma_{z}=1,36 ; \\
\tau_{x y}=0,50 ; & \tau_{y z}=0,69 ; & \tau_{z x}=1,65 .
\end{aligned}
$$

Принимая $\Theta_{x}=\Theta_{y}=45^{\circ}$ и $\Theta_{x}^{\prime}=-45^{\circ}$, точные экспериментальные данные будут следующими:

$$
\begin{array}{llll}
\delta_{\mathrm{H}}=1,67 ; & \delta_{x}=2,43 ; & \delta_{x}^{\prime}=5,12 ; & \delta_{y}=2,71 ; \quad \delta^{*}=6,48 ; \\
\varphi_{\mathrm{H}}=71,5^{\circ} ; & \varphi_{x}=-54,6^{\circ} ; \quad \varphi_{x}^{\prime}=61,3^{\circ} ; \quad \varphi_{y}=30,8^{\circ} .
\end{array}
$$

\section{Таблица 3}

\begin{tabular}{c|c}
\hline $\begin{array}{c}\text { Компонент } \\
\text { напряжения }\end{array}$ & $\varepsilon$ \\
\hline$\sigma_{x}-\sigma_{y}$ & 0,015 \\
$\sigma_{y}-\sigma_{z}$ & 0,058 \\
& $(0,049)$ \\
$\tau_{x y}$ & 0,008 \\
$\tau_{y z}$ & 0,018 \\
& $(0,021)$ \\
$\tau_{z x}$ & 0,015 \\
$\sigma_{x}$ & 0,024 \\
& $(0,024)$ \\
$\sigma_{y}$ & 0,026 \\
& $(0,030)$ \\
$\sigma_{z}$ & 0,043 \\
& $(0,036)$ \\
$\sigma_{1}-\sigma_{3}$ & 0,073 \\
$\sigma_{2}-\sigma_{3}$ & 0,034 \\
$\sigma_{1}$ & 0,059 \\
$\sigma_{2}$ & 0,032 \\
$\sigma_{3}$ & 0,039
\end{tabular}

В рассматриваемом случае при применении универсального метода Е. С. Федорова можно использовать нормальное просвечивание среза.

Предполагаем следующую точность эксперимента:

$\bar{\Delta} \delta_{i}=0,005 \delta_{\max }=0,0256 ; \quad \bar{\Delta} \Theta_{i}=0.5^{\circ}=0,00873 ;$

$\bar{\Delta} \varphi_{i}=1,0^{\circ}=0,0175 ; \bar{\Delta}^{*}=0,05 \delta^{*}=0,324$.

Точность определения углов методом Е. С. Федорова предполагается равной $1^{\circ}, K_{1}=0,25$.

Средние квадратичные ошибки компонентов напряжения, вычисленные изложенным выше методом, представлены в табл. 3.

Как явствует из таблицы, точность определения напряжений является удовлетворительной.

П ри меч ани е. В скобках прнведены величины ошибок для случая, когда для наклонного просвечивання модель поворачивается около осн $\boldsymbol{x}$ на разные углы.

\section{5. Заключение}

Хотя приведенный частный пример не позволяет сделать окончательных выводов относительно точности рассмотренного метода, изложенные соображения позволяют заключить, что метод может оказаться эффективным при полном определении объемного напряженного состояния.

Преимуществом предложенного метода по сравнению с обобщенным методом разности касательных напряжений является независимое определение напряжений в каждой точке. Кроме того, для определения напряжений в одной плоскости модели требуется исследовать только один 
срез. Главные напряжения могут быть произвольно ориентированы относительно плоскости среза. Существенным является также то, что абсолютные разности хода приходится измерять только при нормальном просвечивании среза.

Что касается измерения абсолютных разностей хода, то методика и аппаратура для этого хорошо разработаны, причем в последнее время измерение абсолютных разностей хода применяется в весьма сложных случаях, например, при исследовании пластинок методом слоистых моделей $\left[{ }^{10}\right]$. Отметим, что Б. М. Зуевым $\left.{ }^{11}\right]$ сконструирован интерферометр, который предназначен также для исследования замороженных срезов.

\title{
ЛИТЕРА Т У Р А
}

1. В. М. К раснов, О решении пространственной задачи теории упругости оптическим методом, Уч. зап. ЛГУ. Серия мат. наук, № 44, 1939.

2. A. Kuske, Das Kunstharz Phenolformaldehyd in der Spannungsoptik, Forschung auf dem Gebiete des Ingenieurwesens, 9, 1938.

3. M. M. Frocht, R. G u e r n sey, Studies in Three-Dimensional Photoelasticity, Proc. First U. S. National Congress of Appl. Mech., Publ. Am. Soc. Mech. Engrs., N-Y, 1952.

4. H. T. Jessop, The Determination of the Separate Stresses in Three-Dimensional Stress Investigations by the Frozen Stress Method, J. of Scient. Instr. and Physics in Industry, 26, No. 1, 1949.

5. H. H. R a cké, Räumliche Spannungsbestimmung an durchsichtigen Modellen mittels der Deformation kleiner Kugeleinschlüsse, Forschung auf dem Gebiete des Ingenieurwesens, 21, Nr. 5, 1955.

6. R. P l e ch a t a, Přispévek $\mathrm{k}$ fotoelasticimetrické separaci hlavnich napěti $\mathrm{v}$ prostorových problémech, Rozpravy Ceskoslovenské Akadenie Vèd, Řada TV, Ročnik 64, sešit $3,1954$.

7. Х. К. А бен, О применении метода наклонного просвечивания в фотоупругости, Изв. АН ЭССР. Серия техн. и физ.-мат. наук, т. IX, № 1, 1960.

8. H. T. Jess o p, M. K. W ells, The Determination of the Principal Stress Differences at a Point in a Three-Dimensional Photoelastic Model, British Journal of Applied Physics, 1, No. 7. 1950.

9. R. Fle ury, F. Z a n d m a n, Emploi de la platine Federoff pour la détermination des trois différences des tensions principales dans les modèles figés, Comptes Rendus Acad. Sci., 239, No. 3, 1954.

10. B. Gilg, Experimentelle und theoretische Untersuchungen an dünnen Platten, Publications du laboratoire de photoélasticité, Ecole Polytechnique Fédérale, Zurich, 1952.

11. Б. М. Зуев, Разделение главных или квазиглавных напряжений в прозрачных моделях оптическим методом. Изв. Казанского филиала АН СССР. Серия физ.-мат. и техн. наук, вып. 12, 1958.

Институт энергетики

Академии наук Эстонской ССР

Поступнла в редакцию

10. VI 1959

\section{RUUMILISE PINGEOLUKORRA TAIELIKUST MĂARAMISEST FOTOELASTSUSMEETODIL}

\author{
H. Aben, \\ tehnikakandidaat
}

Resümee

Ruumilise pingeolukorra täielikuks määramiseks külmutusmeetodil koormatud mudelist välja lōigatud plaadis esitatakse artiklis puhtpolarisatsioonoptiline meetod, mis baseerub kaldvalgustusmeetodil ning ristvalgustamisel esinevate absoluutsete faasinihete mõōtmisel. Hariliku polariskoobi kasutamisel tuleb lisaks ristvalgustamisel esinevate absoluutsete faasinihete mõōtmisele määrata faasinihe ning quasiisokliini parameeter ristvalgustamisel 
ja kahel kaldvalgustamisel. Kasutades peapingete suundade leidmiseks J. S. Fjodorovi universaalset meetodit, tuleb määrata absoluutsed faasinihked ristvalgustamisel ning faasinihe ja quasiisokliini parameeter ühel läbivalgustamisel. Analüüsitakse ka meetodi täpsust.

\section{Eesti NSV Teäduste Akadeemia Energeetika Instituut}

Saabus toimetusse

10. VI 1959

\section{ON THE COMPLETE DETERMINATION OF THE THREE-DIMENSIONAL STATE OF STRESS BY PHOTOELASTIC METHODS}

\section{H. Aben}

\section{Summary}

For the complete determination of stress in frozen slices a purely photoelastic method has been developed, which involves the use of the oblique incidence method and the measurement of the absolute phase differences by normal incidence. If a common polariscope is used, fringe orders and parameters of isoclinics by normal and two oblique incidences in addition to absolute phase differences by normal incidence are to be determined. If the directions of principal stresses are determined by the universal stage of E. Fedorov, fringe order and parameter of isoclinic by a single incidence as well as absolute phase differences by normal incidence are to be determined. The accuracy of the method is studied.

Academy of Sciences of the Estonian S.S.R., Institute of Energetics

Received

June 10th, 1959 